\title{
Convex modeling for optimal battery sizing and control of an electric variable transmission powertrain
}

\author{
Zetao $\mathrm{Ma}^{1}$, Nikolce Murgovski ${ }^{2}$, Bo Egardt ${ }^{2}$, and Shumei Cui ${ }^{1, *}$ \\ ${ }^{1}$ School of Electrical Engineering and Automation, Harbin Institute of Technology, 15001 Harbin, China \\ ${ }^{2}$ Department of Electrical Engineering, Chalmers University of Technology, 41296 Gothenburg, Sweden
}

Received: 28 August 2018 / Accepted: 17 December 2018

\begin{abstract}
This paper provides convex modeling steps for the problem of optimal battery sizing and energy management of a plug-in hybrid electric vehicle with an electric variable transmission. Optimal energy management is achieved by a switched model control, with driving modes identified by the engine on/off state. In pure electric mode, convex optimization is used to find the optimal torque split between two electric machines, in order to maximize powertrain efficiency. In hybrid mode, optimization is performed in a bilevel program. One level optimizes speed of a compound unit that includes the engine and electric machines. Another level optimizes the power split between the compound unit and the battery. The proposed method is used to minimize the total cost of ownership of a passenger vehicle for a daily commuter, including costs for battery, fossil fuel and electricity.
\end{abstract}

\section{Introduction}

Hybrid Electric Vehicles (HEVs) are being considered a convenient intermediate product in the conversion process from conventional to pure electric vehicles, due to their compromise on cost, fuel consumption and driving range. With the improved performance and reduced cost of battery pack, Plug-in HEV (PHEV), which is $\mathrm{HEV}$ but equipped with a larger battery and a charging connector, are also becoming popular solutions. Different from $\mathrm{HEV}, \mathrm{PHEV}$ can get recharged from the electricity grid and thus have longer driving range in pure electric mode and can achieve lower fuel consumption and emissions. PHEV is especially suitable for daily commuting. For instance, a household PHEV could be recharged during the night and deplete the charge in the daily driving routes.

HEV and PHEV powertrains possess an Internal Combustion Engine (ICE), one or several Electric Machines (EMs) and a battery. Depending on how the ICE and EMs propel the vehicle, HEV and PHEV powertrains could be classified into series, parallel and series-parallel architectures. Series-parallel powertrains currently dominate the hybrid vehicle sector due to their remarkable fuel economy improvement [1]. Recently, series-parallel powertrains equipped with an Electric Variable Transmission (EVT) have been widely studied $[2-5]$. These powertrains function

\footnotetext{
* Corresponding author: cuism@hit.edu.cn
}

similarly as the well known THS II HEV powertrain used in Prius [6], i.e. they involve an ICE and two EMs. However, instead of planetary gear sets, the EVT powertrain uses a double rotor machine (typically considered as two EMs in one package) to mechanically decouple the engine from the wheels. The engine is still able to deliver part of its power to the final drive to propel the vehicle, by the use of magnetic coupling.

When designing PHEV powertrains, typical optimization problems involve battery sizing and design of Energy Management Strategy (EMS). Usually, these two problems are coupled to each other and should thus be optimized simultaneously. Such optimization could be performed in different ways, but among the most common methods is the nested optimization approach, where battery capacity is gridded into several sizes and then for each size the EMS is optimized.

EMS could be designed in two major ways; heuristic rule-based formulation or optimization approaches [7-14]. For component sizing problems, optimization approaches are the preferred choice, since the optimal results provide objective benchmark for comparing different powertrains. From the proposed EMS optimization approaches, Dynamic Programming (DP) is the most commonly used. However, DP is highly demanding on computation power, especially if high accuracy is required. Furthermore, the computation complexity of DP increases exponentially with the number of state variables [15]. Regardless of these deficiencies, DP is still widely used due to its capability of handling mix-integer problems, 
which in the HEV context typically includes engine on/off and gearshift control [16].

Another EMS optimization approach that has been shown to require less computational power, relies on Pontryagin's Minimum Principle (PMP) [12, 17-19]. However, when applying PMP in the HEV problems, it is assumed that the battery size is large enough to guarantee a constant Lagrange multiplier, while searching for the optimal control that does not activate battery energy limits [12]. Another limitation of PMP is the inability of handling integer state variables. The latter deficiency is typically addressed by combining PMP and DP (DP-PMP) to iteratively solve the mixed-integer control problems [19].

Convex optimization has also been proposed to solve the optimization problems in HEVs [20-29]. By exploiting convexity of the powertrain models, the powertrain sizing and EMS problems could be efficiently solved using publicly available solvers for convex programs. Most importantly, different from DP or PMP, convex optimization allows battery sizing to be solved simultaneously with the EMS optimization [23-25]. Convex optimization, however, cannot solve integer problems. Approaches that overcome this limitation rely on a synergy between convex optimization and DP [21, 29], or convex optimization and PMP [22]. Another difficulty is that convex optimization often requires convex modeling steps that are specifically tailored to different powertrain configurations. Convex modeling steps have currently been shown for powertrains in series configuration [21, 24], parallel configuration [24, 25], configurations that involve a planetary gear [30] or continuous variable transmission [23]. To the best of the author's knowledge, convex optimization has not yet been applied to EVT powertrains.

This paper extends previous studies by providing convex modeling steps for the problem of optimal battery sizing and control of a series-parallel PHEV powertrain equipped with an EVT. The optimization cost is formulated as the total cost of ownership, which includes operational cost for fuel and electricity consumption, and component cost for the battery pack. Similarly to the optimal EMS of a planetary gear powertrain [30], we show that the EVT powertrain can be optimally controlled by a bilevel optimization, where one level optimizes the speed of a compound unit including the ICE and EMs, and another layer optimizes power split between the compound unit and the battery. Different from the approach in [30], we show that the optimal EMS of the EVT powertrain involves a switched model control. In a hybrid mode of operating, when the engine is on, power split is decided between the compound unit and the battery. In pure electric mode, when the engine is off, torque split is decided between the two EMs, which further improves performance, compared to the typical case when only a single EM drives the vehicle [5]. Moreover, we show that for a given driving cycle and engine on/off control, convex optimization can be used to simultaneously optimize both the EMS and the battery size. Several case studies are provided that show the dependence of the optimal battery size on the battery discharge strategy and the validity of the proposed approach compared to a benchmark solution obtained by DP.
The remainder of this paper is organized as follows. Section 2 provides the modeling details of the powertrain and formulates the studied optimization problem. Section 3 presents the convex modeling steps, which is the main contribution of this paper. Case studies are provided in Section 4. Conclusion and future work are discussed in Section 5 .

\section{EVT powertrain and problem statement}

This section introduces a mathematical model of the EVT powertrain. It introduces the daily driving cycle on which the powertrain is evaluated and formulates the studied optimization problem.

\subsection{EVT powertrain}

EVT is a compact double rotor machine which can be divided into an ordinary Electric Machine (EM2) and a dual rotor Electric Machine (EM1). In the EVT HEV powertrain, the ICE crank shaft is connected to the EVT inner rotor, while the input side of final drive is connected to the outer rotor. Between the crank shaft and the inner rotor, a brake is placed that can be used to immobilize the inner rotor. Due to the double rotor design, the EVT can function as an energy converter in the HEV powertrain and realize power split, like the Prius Toyota Hybrid System [6], but without planetary gear sets. The studied EVT plug-in HEV powertrain is illustrated in Figure 1.

The EVT powertrain can operate in three different modes, detailed in Table 1. In pure electric mode, the brake is engaged and EM1 together with EM2 can propel the driveline through the outer rotor. In starter mode, the brake is disengaged and EM1 cranks the ICE until reaching idling speed, before the engine is turned on. The brake can be disengaged even in hybrid electric mode, to allow the engine to be decoupled from the wheels and thus be operated at its optimum efficiency. The EVT powertrain does not provide a mechanical connection between the ICE and the final drive, so the powertrain cannot be operated in a pure combustion mode. Instead, the ICE torque has to be delivered through the interaction with EM1. Typically, under low vehicle speed the ICE drives EM1 as a generator, while EM2 works as a prime motor. Under high vehicle speed, EM2 is operated as a generator, while EM1 functions as the prime motor.

Since the motion dynamics of the EVT are much faster than the battery dynamics, they have been excluded from the battery sizing problem discussed below. Similarly, the starter mode of the powertrain is not discussed further in this paper.

\subsection{Longitudinal dynamics of the powertrain}

In the EVT powertrain, EM1 and EM2 directly drive the vehicle in both electric and hybrid mode. Then the powertrain should satisfy the motion relation

$$
T_{\mathrm{d}}(t)+T_{\mathrm{brk}}(t)=T_{1}(t)+T_{2}(t),
$$




$$
\omega_{2}(t)=\frac{i_{\mathrm{f}}}{r} v(t)
$$

where $T_{1}$ and $T_{2}$ are the EM1 and EM2 torques, respectively, $T_{\mathrm{brk}}$ is the braking torque from the mechanical brakes and $\omega_{2}$ is the EM2 speed. The demanded torque at the input side of the final drive, $T_{\mathrm{d}}$, can be described as

$$
\begin{aligned}
T_{\mathrm{d}}(t)= & \frac{r \eta_{\mathrm{f}}^{-\operatorname{sgn}\left(T_{\mathrm{d}}\right)}}{i_{\mathrm{f}}}\left(\frac{\rho C_{\mathrm{d}} v^{2}(t)}{2}+m g f \cos \theta(t)\right. \\
& +m g \sin \theta(t)+m \dot{v}(t)),
\end{aligned}
$$

where $r$ is the wheel radius, $i_{\mathrm{f}}$ is the ratio of the final drive, $\eta_{\mathrm{f}}$ is the transmission efficiency of the final drive, $\rho$ is the air density, $g$ is the acceleration of gravity, $f$ is the friction coefficient, $\theta$ represents the road slope, $v$ is the vehicle speed, and $m$ is the total vehicle mass. The vehicle mass can be expressed as

$$
m=m_{\mathrm{v}}+m_{\mathrm{b}},
$$

where $m_{\mathrm{b}}$ is the mass of the battery pack and $m_{\mathrm{v}}$ is the remaining vehicle mass.

The sgn function in (3) returns the sign of $T_{\mathrm{d}}$, which is a time dependent signal. In order of improving readability by reducing the number of nested parentheses, the explicit dependence on time will not be shown for signals that are input arguments to functions.

\subsubsection{Pure electric mode}

In pure electric mode, the inner rotor of the EVT is immobilized. The powertrain could either be driven by the dual motors EM1 and EM2, or only by EM2 when $T_{1}=0$. When the EM1 is on, the speed relation of EM1 and EM2 should satisfy

$$
\omega_{1}(t)=\omega_{2}(t),
$$

where $\omega_{1}$ is the EM1 speed.

\subsubsection{Hybrid electric mode}

In hybrid electric mode, part of the ICE power propels the driveline through the torque balance with EM1, while the remaining part is turned into electric energy due to the speed difference of the two rotors. That is to say, the ICE torque $T_{\mathrm{e}}$ should balance the EM1 torque, while the EM1 speed is expressed at the speed difference between the two rotors:

$$
\begin{gathered}
T_{\mathrm{e}}(t)=T_{1}(t), \\
\omega_{1}(t)=\omega_{2}(t)-\omega_{\mathrm{e}}(t) .
\end{gathered}
$$

\subsection{Power balance}

The powertrain should always satisfy the electric power balance among the battery pack, EM1 and EM2,

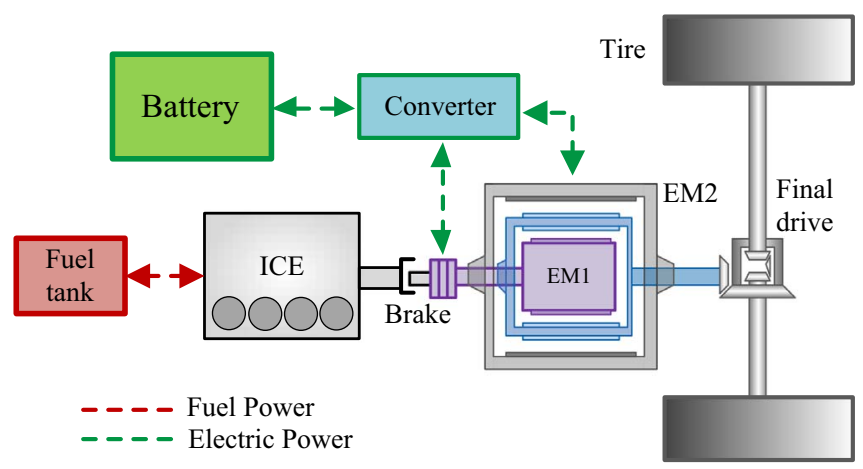

Fig. 1. Architecture of the EVT plug-in HEV powertrain. The powertrain includes an Internal Combustion Engine (ICE) and a double rotor machine which can be regarded as an ordinary Electric Machine (EM2) and a dual rotor Electric Machine (EM1). The brake between the crank shaft and the inner rotor can be used to immobilize the inner rotor.

Table 1. Operational modes of the powertrain.

\begin{tabular}{lcccc}
\hline Modes & ICE & Brake & EM1 & EM2 \\
\hline Pure electric mode & Off & Engaged & On & On \\
Starter mode & Off & Disengaged & On & On \\
Hybrid electric mode & On & Disengaged & On & On \\
\hline
\end{tabular}

$$
\begin{aligned}
P_{\mathrm{b}}(t)= & T_{1}(t) \omega_{1}(t)+B_{1}(t)+T_{2}(t) \omega_{2}(t)+B_{2}(t) \\
& +B_{\mathrm{b}}(t)+P_{\text {aux }},
\end{aligned}
$$

where $P_{\mathrm{b}}$ is the total power output of the battery pack, $B_{\mathrm{b}}$ is the battery power loss, $B_{1}$ is the EM1 power loss, $B_{2}$ is the EM2 power loss and $P_{\text {aux }}$ is the auxiliary power which is considered as a constant.

By substituting (1), (6) and (7) into (8), the power balance can also be written as

$$
\begin{aligned}
P_{\mathrm{b}}(t)= & -T_{\mathrm{e}}(t) \omega_{\mathrm{e}}(t)+B_{1}(t)+B_{2}(t) \\
& +\left(T_{\mathrm{d}}(t)+T_{\mathrm{brk}}(t)\right) \omega_{2}(t)+B_{\mathrm{b}}(t)+P_{\text {aux }} .
\end{aligned}
$$

In pure electric mode, the engine is turned off and the brake at the crank shaft is engaged, enforcing $T_{\mathrm{e}}=0$ and $\omega_{\mathrm{e}}=0$ in (9).

\subsection{Daily driving routes}

In this study the main usage of the plug-in vehicle is considered to be routine commuting. Since it is difficult to predict and model the traffic conditions over the vehicle's lifespan, we adopt a representative daily route as the driving cycle. The commuter route is driven over 50 times and then the logged data is trained to a typical representative daily cycle. This route goes from the suburb of Kungsbacka to an industrial facility on the outskirts of Gothenburg in Sweden, along a motorway. It is assumed that the vehicle could only get charged at home during nighttime. The driving cycle, including the typical speed and altitude profile from home to work and vice versa, is shown in Figure 2. 


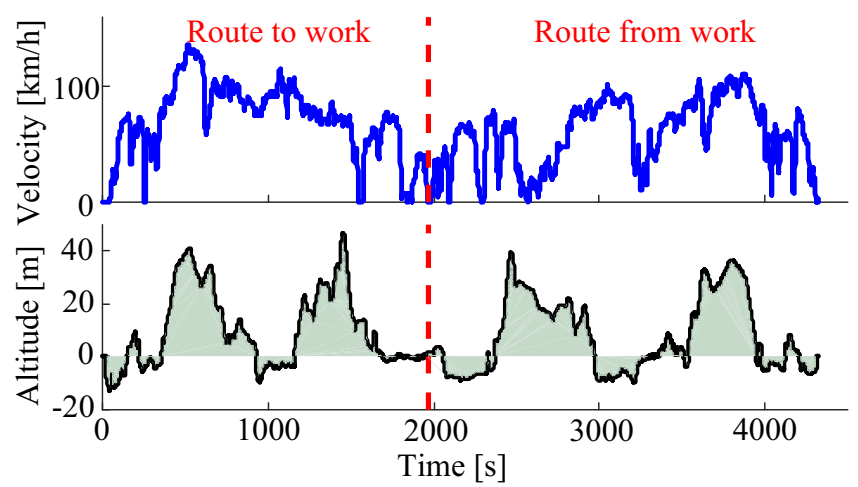

Fig. 2. Profile of the daily driving cycle, depicted as speed and altitude as a function of time. The daily cycle is divided into two segments, one is the route to work and the other is the route from work. The battery is charged only at nighttime.

\subsection{ICE model}

Willans line are used to depict the engine model, as shown in Figure 3. The ICE fuel power is fitted into quadratic functions of its torque, as

$$
P_{\mathrm{f}}(t)=e_{\mathrm{on}}(t) a_{0}\left(\omega_{\mathrm{e}}\right)+a_{1}\left(\omega_{\mathrm{e}}\right) T_{\mathrm{e}}(t)+a_{2}\left(\omega_{\mathrm{e}}\right) T_{\mathrm{e}}^{2}(t),
$$

where $a_{0}, a_{1}$ and $a_{2}$ depend on the engine speed, with $a_{0}$, $a_{1}, a_{2} \geq 0, \forall \omega_{\mathrm{e}}$. Alternatively, it is possible to express fuel power as

$$
P_{\mathrm{f}}(t)=\omega_{\mathrm{e}}(t) T_{\mathrm{e}}(t)+B_{\mathrm{e}}(t)
$$

where

$$
\begin{aligned}
B_{\mathrm{e}}(t)= & e_{\mathrm{on}}(t) a_{0}\left(\omega_{\mathrm{e}}\right)+\left(a_{1}\left(\omega_{\mathrm{e}}\right)-\omega_{\mathrm{e}}\right) T_{\mathrm{e}}(t) \\
& +a_{2}\left(\omega_{\mathrm{e}}\right) T_{\mathrm{e}}^{2}(t),
\end{aligned}
$$

is the ICE power loss.

The engine on/off signal $e_{\mathrm{on}}$ is

$$
e_{\mathrm{on}}(t)= \begin{cases}1, & \text { if ICE on } \\ 0, & \text { otherwise }\end{cases}
$$

Since in the EVT powertrain the ICE can be started by the EM1, the ICE is only allowed to work at the region above the idling speed.

\subsection{EM models}

Depending on the sign of speed and torque, electric machines may operate in four different quadrants. The quadrants, from one to four, are enumerated here as follows: (1) positive speed and positive torque, (2) negative speed and positive torque, (3) negative speed and negative torque and (4) positive speed and negative torque. In pure electric mode, the EM1 works in the first quadrant for driving or in the forth quadrant for regenerative braking. In hybrid mode, the EM1 works in the first or second quadrant to balance the ICE torque. EM2 operates in the first or forth quadrant.

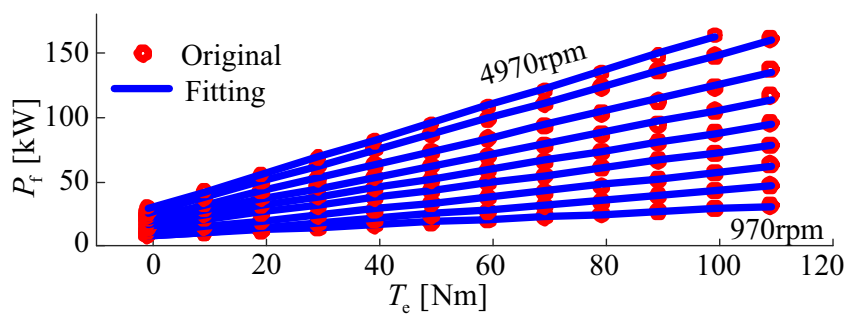

Fig. 3. Original data and fitted model of the ICE fuel power, as a function of torque and speed. Each line represents a constant engine speed. Detailed specification of the engine is listed in Table A1.

Similar to the ICE, the electric machines are also modeled with Willans lines. Their power loss within the different modes can be expressed as

$$
\begin{aligned}
& B_{1}(t)=b_{0}\left(\omega_{1}\right)+b_{1}\left(\omega_{1}\right) T_{1}(t)+b_{2}\left(\omega_{1}\right) T_{1}^{2}(t), \\
& B_{2}(t)=c_{0}\left(\omega_{2}\right)+c_{1}\left(\omega_{2}\right) T_{2}(t)+c_{2}\left(\omega_{2}\right) T_{2}^{2}(t),
\end{aligned}
$$

where $B_{1}, B_{2}$ are the EM1 and EM2 power losses, respectively, and $b_{0}, b_{1}, b_{2}$, with $b_{0}, b_{1}, b_{2} \geq 0, \forall \omega_{1}$ and $c_{0}, c_{1}, c_{2}$, with $c_{0}, c_{1}, c_{2} \geq 0, \forall \omega_{2}$, are speed depended coefficients. The EM1 and EM2 models are depicted in Figures 4 and 5 , respectively.

\subsection{Battery model}

A simplified open-circuit model is used to approximate the lithium-ion battery pack. It comprises $n_{\mathrm{b}}$ identical cells whose open-circuit voltage $V_{\mathrm{b}}$ and cell's internal resistance $R_{\mathrm{b}}$ are considered as constant. It is reasonable and accurate enough to model the battery cell in this way if the cell is only operated in a limited state of charge region, as illustrated in Figure 6. Assuming that the battery cells' dynamics do not change, the battery pack can be modeled as

$$
\begin{gathered}
P_{\mathrm{b}}(t)=n_{\mathrm{b}} V_{\mathrm{b}} i_{\mathrm{b}}(t), \\
\dot{E}_{\mathrm{b}}(t)=-P_{\mathrm{b}}(t),
\end{gathered}
$$

where $i_{\mathrm{b}}$ is the cell current and $E_{\mathrm{b}}$ is the total pack energy described by

$$
E_{\mathrm{b}}(t)=n_{\mathrm{b}} Q_{\mathrm{c}} V_{\mathrm{b}} \operatorname{soc}(t),
$$

where $Q_{\mathrm{c}}$ is the cell capacity and soc is the state of charge.

The loss of the battery pack can be expressed as quadratic function of the internal power,

$$
B_{\mathrm{b}}(t)=n_{\mathrm{b}} i_{\mathrm{b}}^{2}(t) R_{\mathrm{b}}=\frac{R_{\mathrm{b}}}{n_{\mathrm{b}} V_{\mathrm{b}}^{2}} P_{\mathrm{b}}^{2}(t) .
$$

The incremental mass of the pack scales linearly with the number of cells, i.e.

$$
m_{\mathrm{b}}=m_{\mathrm{b} 0} n_{\mathrm{b}}
$$




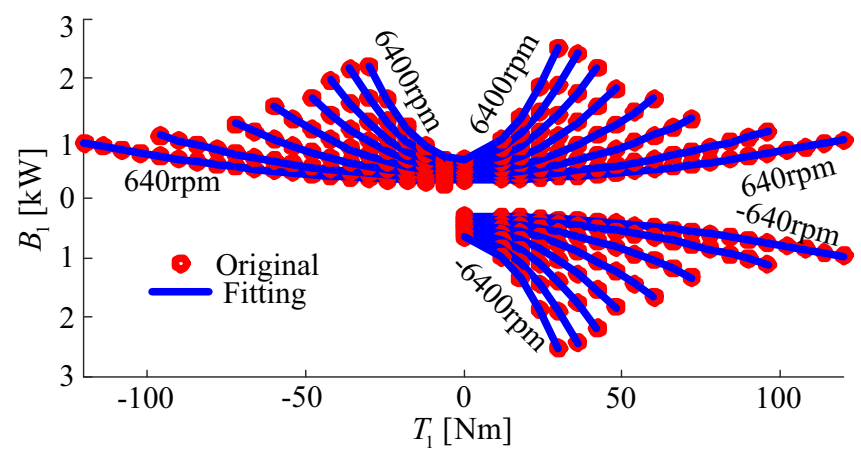

Fig. 4. Original data and fitted model of the EM1 power loss. The EM1 operates in the first and forth quadrants (positive speed) for engine starting/driving and generative braking in pure electric mode. In hybrid mode, EM1 works in first and second quadrants (positive torque) to balance the engine torque. Detailed EM1 specification is listed in Table A1.

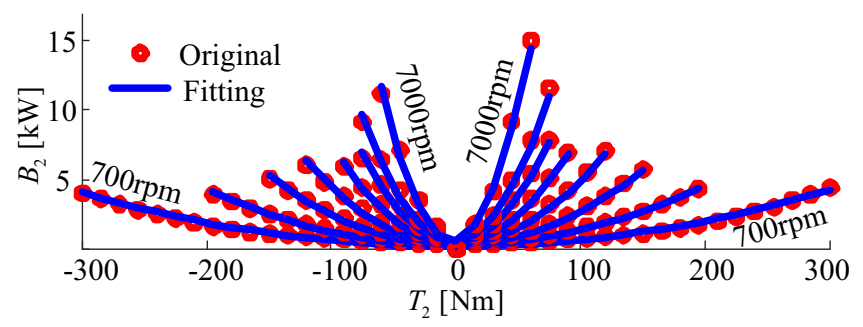

Fig. 5. Original data and fitted model of the EM2 power loss. The EM2 operates in the first and forth quadrants (positive speed) for driving and generative braking. Detailed EM2 specification is listed in Table A1.

The relations (16)-(19) are valid regardless of how battery cells are connected. Thus, after optimizing the pack size $n_{\mathrm{b}}$, the cells can be configured in suitable series and j parallel branches.

\subsection{Optimization problem statement}

The aim of this paper is to minimize the total cost of vehicle ownership, including operational and component costs. The operational cost includes the electricity and gasoline cost. The components cost includes only the cost of the battery pack, since the other parts of the vehicle remain the same and are thus excluded from the optimization. The battery pack is assumed to have good durability and no replacements are required during the vehicle lifespan.

The battery cost is daily averaged during the vehicle's lifespan. Considering a yearly interest rate, the battery cost can be calculated by

$$
C_{\text {bat }}\left(n_{\mathrm{b}}\right)=\frac{d_{\mathrm{r}}}{d_{\mathrm{y}} y_{\mathrm{v}}}\left(1+r_{\mathrm{int}} \frac{1+y_{\mathrm{v}}}{2}\right) p_{\mathrm{bat}} n_{\mathrm{b}}
$$

where $p_{\text {bat }}$ is the price of one single battery cell, $C_{\text {bat }}$ is the battery cost in a monetary currency. The division with the average driving distance in one year $d_{\mathrm{y}}$ and the vehicle lifetime in years $y_{\mathrm{v}}$, provides a battery price per $\mathrm{km}$, which

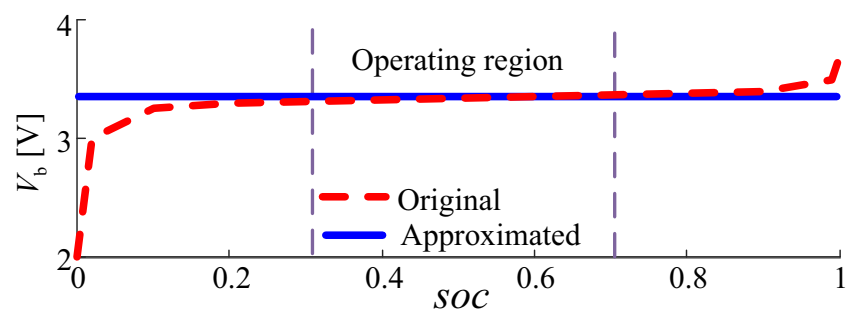

Fig. 6. Original and approximated battery cell open circuit voltage. The battery state of charge is allowed to vary within $0.3-0.7$, in which the open circuit voltage can be considered as constant. Detailed battery parameters are listed in Table A1.

after multiplying with the length of the driving cycle $d_{\mathrm{r}}$ provides the battery price in a monetary currency for the specific daily driving cycle. The part in parentheses depicts depreciation of value, where $r_{\text {int }}$ is a yearly interest rate.

Assuming that the battery is charged at night, as often as possible, the electricity cost can be expressed as

$$
C_{\mathrm{el}}\left(E_{\mathrm{b}}\right)=p_{\mathrm{el}}\left(E_{\mathrm{b}}\left(t_{0}\right)-E_{\mathrm{b}}\left(t_{\mathrm{f}}\right)\right),
$$

where $p_{\mathrm{el}}$ is the electricity price expressed as a monetary currency per energy. The difference between initial and final battery energy $\mathrm{E}_{\mathrm{b}}(0)-E_{\mathrm{b}}\left(t_{\mathrm{f}}\right)$ describes the consumed electric energy over the driving cycle, which after multiplying with $p_{\mathrm{el}}$ provides the electricity consumption in a monetary currency. Both the initial and final energy values are considered as optimization variables and the optimization program, described below, is let to find their best values.

Similarly, the gasoline cost can be computed as

$$
C_{\text {fuel }}\left(\omega_{\mathrm{e}}, T_{\mathrm{e}}, e_{\mathrm{on}}\right)=\frac{p_{\mathrm{f}}}{q_{h}} \int_{t_{0}}^{t_{\mathrm{f}}} P_{\mathrm{f}}\left(\omega_{\mathrm{e}}, T_{\mathrm{e}}, e_{\mathrm{on}}\right) \mathrm{d} t,
$$

where $p_{\mathrm{f}}$ is the gasoline price expressed as a monetary currency per liter, and $q_{h}$ is the heating value of gasoline in energy per liter.

Using the above mathematical models, along with the physical limits of the components, the optimization problem can be summarized as

$$
\begin{gathered}
\min C_{\text {bat }}\left(n_{\mathrm{b}}\right)+C_{\mathrm{el}}\left(E_{\mathrm{b}}\right)+C_{\text {fuel }}\left(\omega_{\mathrm{e}}, T_{\mathrm{e}}, e_{\mathrm{on}}\right) \\
\text { subject to }:(1)-(19) \\
n_{\mathrm{b}} \geq 0 \\
T_{\mathrm{brk}}(t) \geq 0 \\
P_{\mathrm{b}}(t) \in V_{\mathrm{b}} n_{\mathrm{b}}\left[I_{\mathrm{bmin}}, I_{\mathrm{bmax}}\right] \\
E_{\mathrm{b}}(t) \in\left[\operatorname{soc}_{\min }, \operatorname{soc}_{\text {max }}\right] n_{\mathrm{b}} Q_{\mathrm{c}} V_{\mathrm{b}} \\
\omega_{1}(t) \in\left[\omega_{1 \text { min }}, \omega_{1 \text { max }}\right] \\
\omega_{2}(t) \in\left[\omega_{2 \min }, \omega_{2 \max }\right]
\end{gathered}
$$




$$
\begin{gathered}
\omega_{\mathrm{e}}(t) \in\left[\omega_{\mathrm{emin}}, \omega_{\mathrm{emax}}\right] \\
T_{1}(t) \in\left[T_{1 \min }\left(\omega_{1}\right), T_{1 \max }\left(\omega_{1}\right)\right] \\
T_{2}(t) \in\left[T_{2 \min }\left(\omega_{2}\right), T_{2 \max }\left(\omega_{2}\right)\right] \\
T_{\mathrm{e}}(t) \in e_{\mathrm{on}}(t)\left[T_{\mathrm{emin}}\left(\omega_{\mathrm{e}}\right), T_{\mathrm{emax}}\left(\omega_{e}\right)\right] .
\end{gathered}
$$

In $(23)$, the battery energy $E_{\mathrm{b}}$ is chosen as the dynamic state and the battery power $P_{\mathrm{b}}$, engine on/off signal $e_{\mathrm{on}}$, engine torque $T_{\mathrm{e}}$ and the engine speed $\omega_{\mathrm{e}}$ as the control signals. The optimization objective is to find the optimal control signals and the optimum parameter $n_{\mathrm{b}}$ that minimize the total cost of vehicle ownership, while satisfying the constraints.

\section{Convex modeling}

This section introduces the convex modeling of the EVT powertrain. To formulate the above optimization problem into a convex form, the engine on/off control is depopulated to a separate subproblem to avoid mix-integer programming, which will be discussed later, in Section 3.3. For a given engine on/off sequence, the convex formulation is separated into pure electric and hybrid mode of operation. To ensure convexity of the battery pack model, (16)-(19), the integer cells number $n_{\mathrm{b}}$ is relaxed to a real value, which can be rounded to the nearest integer after the optimization is finished. In addition, it is initially assumed that the total vehicle mass does not visibly change with battery sizing. The sensitivity on battery mass will be discussed later, in Section 4.

\subsection{Pure electric driving mode}

Consider the situation when the engine is turned off. In pure electric mode, electric machine torques are the only control signals to propel the vehicle, while their speed is entirely determined by the driving cycle. To minimize the energy dissipation of the powertrain in this mode, the two machines can be fully utilized to obtain optimal powertrain efficiency. In this case, due to the convex models of the electric machines in (14) and (15), convex optimization can be used to find the optimal torque distributions between these two machines, by relaxing (9) into inequality

$$
\begin{gathered}
T_{1}(t)+T_{2}(t)=T_{\mathrm{d}}(t)+T_{\mathrm{brk}}(t), \\
P_{\mathrm{b}}(t) \geq\left(T_{\mathrm{d}}(t)+T_{\text {brk }}(t)\right) \omega_{2}(t)+B_{1}\left(\omega_{1}, T_{1}\right) \\
+B_{2}\left(\omega_{2}, T_{2}\right)+B_{\mathrm{b}}\left(P_{\mathrm{b}}, E_{\mathrm{b}}, n_{\mathrm{b}}\right)+P_{\mathrm{aux}},
\end{gathered}
$$

where $T_{\mathrm{d}}$ and $\omega_{1}=\omega_{2}$ are fully described by the driving cycle. The engine power term $-T_{\mathrm{e}} \omega_{\mathrm{e}}$ in (9) is, in this case, set to zero, since the engine is turned off.

By relaxing (9) to the inequality (24b), outer approximation is performed which allows the convex optimization to choose solutions that are not only on the function, but also in its epigraph, thus allowing additional power losses than those necessary to deliver the required electrical power. It can be logically reasoned that the optimal solution will satisfy (9) with equality, since otherwise energy is wasted unnecessarily. Then, the solution of the relaxed problem is identical to the solution of the non-relaxed problem. A rigorous proof that this relaxation does not change the optimal solution can be found in [31].

To compare the results later in simulations, the situation is also formulated in which the powertrain is only driven by EM2 in pure electric mode,

$$
\begin{gathered}
T_{2}(t)=T_{d}(t)+T_{\mathrm{brk}}(t) \\
P_{\mathrm{b}}(t) \geq\left(T_{\mathrm{d}}(t)+T_{\mathrm{brk}}(t)\right) \omega_{2}(t)+B_{2}\left(\omega_{2}, T_{2}\right) \\
+b_{0}^{\prime}\left(\omega_{1}\right)+B_{\mathrm{b}}\left(P_{\mathrm{b}}, E_{\mathrm{b}}, n_{\mathrm{b}}\right)+P_{\mathrm{aux}},
\end{gathered}
$$

where $b_{0}^{\prime}$ is the sum of mechanical loss caused by EM1 bearings and eddy current loss caused by relative movement between the inner and outer rotors, depending on the relative speed of the two rotors.

\subsection{Hybrid driving mode}

In hybrid mode of operation, the engine is considered to be on. Since in this case $e_{\mathrm{on}}=1$ and $e_{\mathrm{on}}$ occurs only in product with other variables, further occurrences of $e_{\text {on }}$ are removed in the rest of this section, for the convenience of readability.

From the problem formulation in (23a), it can be seen that the product of two variables $T_{\mathrm{e}}$ and $\omega_{\mathrm{e}}$ in (9) and the cost function $C_{\text {fuel }}$ are generally not convex and the problem cannot directly be solved by convex optimization. Here, the powertrain model and the optimization problem are reformulated into a convex program.

To begin with, the powertrain model in (9) is simplified by eliminating the signals $T_{2}, \omega_{1}$ and $T_{1}$. This can be achieved by back-substituting the equalities (1), (6) and (7) into $(23 \mathrm{~g})$ and $(23 \mathrm{j})$. Considering that $T_{\mathrm{d}}$ and $\omega_{2}$ are fully determined by the driving cycle, the optimization problem can be reformulated as

$$
\begin{gathered}
\min C_{\mathrm{bat}}\left(n_{\mathrm{b}}\right)+C_{\mathrm{el}}\left(E_{\mathrm{b}}\right)+C_{\text {fuel }}\left(\omega_{\mathrm{e}}, T_{\mathrm{e}}\right) \\
\text { subject to : } \\
\dot{E}_{\mathrm{b}}(t)=-P_{b}(t) \\
P_{b}(t)=-T_{\mathrm{e}}(t) \omega_{\mathrm{e}}(t)+\left(T_{\mathrm{d}}(t)+T_{\mathrm{brk}}(t)\right) \omega_{2}(t)+P_{\mathrm{aux}} \\
+B_{1}\left(\omega_{\mathrm{e}}, T_{2}\right)+B_{2}\left(\omega_{2}, T_{2}\right)+B_{\mathrm{b}}\left(P_{\mathrm{b}}, E_{\mathrm{b}}, n_{\mathrm{b}}\right) \\
E_{\mathrm{b}}(t) \in n_{\mathrm{b}} Q_{\mathrm{c}} V_{\mathrm{b}}\left[\operatorname{soc}_{\min }, \operatorname{soc}_{\max }\right] \\
P_{\mathrm{b}}(t) \in n_{\mathrm{b}} V_{\mathrm{b}}\left[I_{\mathrm{bmin}}, I_{\mathrm{bmax}}\right] \\
n_{\mathrm{b}} \geq 0 \\
T_{\mathrm{brk}}(t) \geq 0 \\
\omega_{\mathrm{e}}(t) \leq \min \left\{\omega_{\mathrm{emax}}, \omega_{2}+\omega_{1 \max }\right\}
\end{gathered}
$$




$$
\begin{gathered}
\omega_{\mathrm{e}}(t) \geq \max \left\{\omega_{\mathrm{emin}}, \omega_{2}+\omega_{1 \min }\right\} \\
T_{\mathrm{e}}(t) \leq \min \left\{T_{\mathrm{emax}}\left(\omega_{\mathrm{e}}\right), T_{1 \max }\left(\omega_{\mathrm{e}}-\omega_{2}\right), T_{\mathrm{d}}(t)\right. \\
\left.+T_{\mathrm{brk}}(t)-T_{2 \min }\left(\omega_{2}\right)\right\} \\
T_{\mathrm{e}}(t) \geq \max \left\{T_{\mathrm{e} \min }\left(\omega_{\mathrm{e}}\right), T_{\mathrm{d}}(t)+T_{\mathrm{brk}}(t)\right. \\
\left.-T_{2 \max }\left(\omega_{2}\right)\right\} .
\end{gathered}
$$

The next step is to eliminate the variable $T_{\mathrm{e}}$, by replacing it with a new variable

$$
P_{\mathrm{c}}(t)=T_{\mathrm{e}}(t) \omega_{\mathrm{e}}(t)-B_{1}(\cdot)-B_{2}(\cdot) .
$$

By applying the power loss models of the electric machines and replacing $T_{2}, T_{1}$ and $\omega_{1}$ with (1), (6) and (7), the expression of $T_{\mathrm{e}}$ can be obtained as

$$
T_{\mathrm{e}}(t)=\frac{-A_{1}(\cdot) \pm \sqrt{A_{1}^{2}(\cdot)-4 A_{0}(\cdot) A_{2}(\cdot)}}{2 A_{2}(\cdot)},
$$

where $A_{0}(\cdot), A_{1}(\cdot)$ and $A_{2}(\cdot)$ are functions of $\omega_{\mathrm{e}}, P_{\mathrm{c}}, \omega_{2}$ and $T_{\mathrm{d}}$, i.e.

$$
\begin{aligned}
& A_{0}(\cdot)=b_{0}\left(\omega_{\mathrm{e}}, \omega_{2}\right)+c_{0}\left(\omega_{2}\right)+c_{1}\left(\omega_{2}\right) T_{d}(t) \\
& +c_{2}\left(\omega_{2}\right) T_{\mathrm{d}}^{2}(t)+P_{\mathrm{c}}(t) \\
& A_{1}(\cdot)=b_{1}\left(\omega_{\mathrm{e}}, \omega_{2}\right)-c_{1}\left(\omega_{2}\right)-2 c_{2}\left(\omega_{2}\right) T_{\mathrm{d}}(t)-\omega_{\mathrm{e}}(t) \\
& A_{2}(\cdot)=b_{2}\left(\omega_{\mathrm{e}}, \omega_{2}\right)+c_{2}\left(\omega_{2}\right) \text {. }
\end{aligned}
$$

In terms of the recent variable change, the engine torque constraints in (26i) and (26j) implicitly transform to

$$
P_{\mathrm{c}} \in\left[P_{\text {cmin }}\left(\omega_{\mathrm{e}}, T_{\mathrm{d}}, \omega_{2}\right), P_{\text {cmax }}\left(\omega_{\mathrm{e}}, T_{\mathrm{d}}, \omega_{2}\right)\right] .
$$

Similarly, the engine fuel power can be expressed as $P_{\mathrm{f}}\left(\omega_{\mathrm{e}}, P_{\mathrm{c}}, \omega_{2}, T_{\mathrm{d}}\right)$, or in terms of the alternative expression $(11)$, it can also be written as

$$
\begin{aligned}
& P_{\mathrm{f}}\left(\omega_{\mathrm{e}}, P_{\mathrm{c}}, \omega_{2}, T_{\mathrm{d}}\right)=P_{\mathrm{c}}+B_{\mathrm{c}}\left(\omega_{\mathrm{e}}, P_{\mathrm{c}}, \omega_{2}, T_{\mathrm{d}}\right) \\
& B_{\mathrm{c}}\left(\omega_{\mathrm{e}}, P_{\mathrm{c}}, \omega_{2}, T_{\mathrm{d}}\right)=B_{1}(\cdot)+B_{2}(\cdot)+B_{\mathrm{e}}(\cdot) .
\end{aligned}
$$

It is clear from (31) that the ICE, EM1 and EM2 can now be considered as a compound unit whose input power, output power and power loss are $P_{\mathrm{f}}(\cdot), P_{\mathrm{c}}(\cdot)$ and $B_{\mathrm{c}}(\cdot)$, respectively. In connection with the electrical power balance (9), it is now evident that the meaning of the compound unit is to generate net power $P_{\mathrm{c}}$ that propels the vehicle together with the battery power $P_{\mathrm{b}}$. The optimal energy management would thus correspond to finding the optimal power split ratio between $P_{\mathrm{c}}$ and $P_{\mathrm{b}}$.

The next convexification step is to eliminate $\omega_{\mathrm{e}}$ from problem (26a). This can be achieved by first rewriting (26a) as a bilevel program

$$
\begin{aligned}
& \min C_{\mathrm{bat}}\left(n_{\mathrm{b}}\right)+ C_{\mathrm{el}}\left(E_{\mathrm{b}}\right)+C_{\text {fuel }}\left(\omega_{\mathrm{e}}^{*}, P_{\mathrm{c}}, \omega_{2}, T_{\mathrm{d}}\right) \\
& \text { subject to : } \\
& \dot{E}_{\mathrm{b}}(t)=-P_{\mathrm{b}}(t) \\
& P_{\mathrm{b}}(t)+P_{\mathrm{c}}(t)=\left(T_{\mathrm{d}}(t)+T_{\mathrm{brk}}(t)\right) \omega_{2}(t)+P_{\mathrm{aux}} \\
&+ B_{\mathrm{b}}\left(P_{\mathrm{b}}, E_{\mathrm{b}}, n_{\mathrm{b}}\right)
\end{aligned}
$$

$$
\begin{gathered}
E_{\mathrm{b}}(t) \in n_{\mathrm{b}} Q_{\mathrm{c}} V_{\mathrm{b}}\left[\operatorname{soc}_{\min }, \mathrm{soc}_{\max }\right] \\
P_{\mathrm{b}}(t) \in n_{\mathrm{b}} V_{\mathrm{b}}\left[I_{\mathrm{b} m i n}, I_{\mathrm{bmax}}\right] \\
n_{\mathrm{b}} \geq 0 \\
T_{\mathrm{brk}}(t) \geq 0 \\
P_{\mathrm{c}}(t) \in\left[P_{\mathrm{cmin}}\left(\omega_{\mathrm{e}}^{*}, T_{\mathrm{d}}, \omega_{2}\right), P_{\mathrm{cmax}}\left(\omega_{\mathrm{e}}^{*}, T_{\mathrm{d}}, \omega_{2}\right)\right] \\
\omega_{\mathrm{e}}^{*}(t)=\arg \min _{\omega_{\mathrm{e}}} P_{\mathrm{f}}\left(\omega_{\mathrm{e}}, P_{\mathrm{c}}, \omega_{2}, T_{\mathrm{d}}\right) \\
\operatorname{subject~to~}: \\
\omega_{\mathrm{e}}(t) \leq \min \left\{\omega_{\mathrm{emax}}, \omega_{2}+\omega_{1 \max }\right\} \\
\omega_{\mathrm{e}}(t) \geq \max \left\{\omega_{\mathrm{emin}}, \omega_{2}+\omega_{1 \min }\right\} \\
P_{\mathrm{c}}(t) \in\left[P_{\mathrm{cmin}}\left(\omega_{\mathrm{e}}, T_{\mathrm{d}}, \omega_{2}\right), P_{\mathrm{cmax}}\left(\omega_{\mathrm{e}}, T_{\mathrm{d}}, \omega_{2}\right)\right] .
\end{gathered}
$$

Although formulation (32) gives identical solution as (26), it does reveal some important properties that were not explicitly visible in (26). For example, the low level problem in (32a) is a completely static program, since the only state in the problem is present in the upper level. Moreover, the low level problem can be solved independently from the top level, since the optimal choice of engine speed does not depend on how the battery is operated in the upper level.

One way to independently solve the low level problem in (32), is by optimizing engine speed for all feasible combinations of the input arguments $P_{\mathrm{c}}, \omega_{2}, T_{\mathrm{d}}$. Let $\mathcal{P}_{\mathrm{c}}, \mathcal{W}_{2}$ and $\mathcal{T}_{\mathrm{d}}$ denote the feasible sets for these arguments, respectively. Then, the optimal engine speed, as a function of the three arguments, can be obtained by solving

$$
\begin{gathered}
\omega_{\mathrm{e}}^{*}\left(P_{\mathrm{c}}, \omega_{2}, T_{\mathrm{d}}\right) \underset{\omega_{\mathrm{e}}}{\operatorname{argmin}} P_{\mathrm{f}}\left(\omega_{\mathrm{e}}, P_{\mathrm{c}}, \omega_{2}, T_{\mathrm{d}}\right) \\
\quad \text { subject to : } \\
\omega_{\mathrm{e}}(t) \leq \min \left\{\omega_{\mathrm{emax}}, \omega_{2}+\omega_{1 \max }\right\} \\
\omega_{\mathrm{e}}(t) \geq \max \left\{\omega_{\mathrm{emin}}, \omega_{2}+\omega_{1 \min }\right\} \\
P_{\mathrm{c}}(t) \in \mathcal{P}_{\mathrm{c}}, \omega_{\mathrm{e}}(t) \in \mathcal{W}_{2}, T_{\mathrm{d}}(t) \in \mathcal{T}_{\mathrm{d}} .
\end{gathered}
$$

To approximately solve problem (33), the sets $\mathcal{P}_{\mathrm{c}}, \mathcal{W}_{2}$ and $\mathcal{T}_{\mathrm{d}}$ and the effective engine speed region can be gridded into finite number of points. The accuracy of the solution would then depend on the grid resolution. For example, consider the case where engine speed range is gridded into $N$ points, $\omega_{\mathrm{e}}=\left[\omega_{\mathrm{e} 1}, \omega_{\mathrm{e} 2}, \ldots, \omega_{\mathrm{e} N}\right]^{\mathrm{T}}$. Applying the engine torque expression (28), the minimum fuel power can be found as

$$
\tilde{P}_{\mathrm{f}}\left(P_{\mathrm{c}}, \omega_{2}, T_{\mathrm{d}}\right)=\min _{\omega_{\mathrm{e}} \in\left\{\omega_{\mathrm{e} 1}, \ldots, \omega_{\mathrm{e} N}\right\}} P_{\mathrm{f}}\left(\omega_{\mathrm{e}}, P_{\mathrm{c}}, \omega_{2}, T_{\mathrm{d}}\right),
$$

for each feasible grid point in $\mathcal{P}_{\mathrm{c}}, \mathcal{W}_{2}$ and $\mathcal{T}_{\mathrm{d}}$. In (34), engine speed is removed and the minimum engine power $\tilde{P}_{\mathrm{f}}$ now only depends on $\left(P_{\mathrm{c}}, \omega_{2}, T_{\mathrm{d}}\right)$, reflecting that for any $\left(P_{\mathrm{c}}, \omega_{2}, T_{\mathrm{d}}\right)$ the compound unit operates at its optimum engine speed. Similarly, the optimal engine speed 
can be replaced in the compound power limits (30), to obtain reduced dimensional limits

$$
\begin{gathered}
\tilde{P}_{\text {cmin }}\left(T_{\mathrm{d}}, \omega_{2}\right)=P_{\text {cmin }}\left(\omega_{\mathrm{e}}^{*}, T_{\mathrm{d}}, \omega_{2}\right) \\
\tilde{P}_{\text {cmax }}\left(T_{\mathrm{d}}, \omega_{2}\right)=P_{\text {cmax }}\left(\omega_{\mathrm{e}}^{*}, T_{\mathrm{d}}, \omega_{2}\right) .
\end{gathered}
$$

After replacing the fuel consumption term $P_{\mathrm{f}}$ with $\tilde{P}_{\mathrm{f}}$ in the objective function, the upper level problem in (32) can be reformulated in a reduced form

$$
\begin{gathered}
\min C_{\mathrm{bat}}\left(n_{\mathrm{b}}\right)+C_{\mathrm{el}}\left(E_{\mathrm{b}}\right)+C_{\text {fuel }}\left(P_{\mathrm{c}}, \omega_{2}, T_{\mathrm{d}}\right), \\
\text { subject to : } \\
\dot{E}_{\mathrm{b}}(t)=-P_{\mathrm{b}}(t), \\
P_{\mathrm{b}}(t)+P_{\mathrm{c}}(t)=\left(T_{\mathrm{d}}(t)+T_{\mathrm{brk}}(t)\right) \omega_{2}(t)+P_{\mathrm{aux}} \\
+B_{\mathrm{b}}\left(P_{\mathrm{b}}, E_{\mathrm{b}}, n_{\mathrm{b}}\right), \\
E_{\mathrm{b}}(t) \in n_{\mathrm{b}} Q_{\mathrm{c}} V_{\mathrm{b}}\left[\operatorname{soc}_{\min }, \operatorname{soc}_{\max }\right], \\
P_{\mathrm{b}}(t) \in n_{\mathrm{b}} V_{\mathrm{b}}\left[I_{\mathrm{b} \min }, I_{\mathrm{bmax}}\right], \\
n_{\mathrm{b}} \geq 0, \\
T_{\mathrm{brk}}(t) \geq 0, \\
P_{\mathrm{c}}(t) \in\left[\tilde{P}_{\mathrm{cmin}}\left(T_{\mathrm{d}}, \omega_{2}\right), \tilde{P}_{\mathrm{cmax}}\left(T_{\mathrm{d}}, \omega_{2}\right)\right] .
\end{gathered}
$$

The minimum fuel power $\tilde{P}_{\mathrm{f}}$ map acquired from (33) is shown in Figure 7 for several choices of $\omega_{2}$ and $T_{\mathrm{d}}$. The net power limits $\tilde{P}_{\text {cmin }}, \tilde{P}_{\text {cmax }}$ maps are shown in Figure 8 . For the given combinations of $\omega_{2}$ and $T_{\mathrm{d}}$, it can be observed that the fuel power appears convex in the net output power $P_{\text {c. }}$. Furthermore, the fuel power is only slightly curved with respect to the compound power $P_{\mathrm{c}}$. Thus, using a least square method the fuel power is fitted by a second order polynomial

$$
\begin{gathered}
\tilde{P}_{\mathrm{f}}\left(\omega_{2}, T_{\mathrm{d}}\right)=k_{0}\left(\omega_{2}, T_{\mathrm{d}}\right)+k_{1}\left(\omega_{2}, T_{\mathrm{d}}\right) P_{\mathrm{c}}(t) \\
+k_{2}\left(\omega_{2}, T_{\mathrm{d}}\right) P_{\mathrm{c}}^{2}(t),
\end{gathered}
$$

where $k_{0}, k_{1}$ and $k_{2}$, with $k_{2} \geq 0, \forall \omega_{2} \in \mathcal{W}_{2}, T_{\mathrm{d}} \in \mathcal{T}_{\mathrm{d}}$, are coefficients depending on $\left(\omega_{2}, T_{\mathrm{d}}\right)$. The approximation of $\tilde{P}_{\mathrm{f}}$ is also shown in Figure 7, where it can be observed that (37) fits well the gridded fuel data. For demanded torque/speed inputs not on the grid points of $\omega_{2}, T_{\mathrm{d}}$, the coefficients' values could be calculated by linear interpolation.

Finally, after relaxing the power balance equation to the inequality

$$
\begin{aligned}
P_{\mathrm{b}}(t)+P_{\mathrm{c}}(t) \geq & \left(T_{\mathrm{d}}(t)+T_{\mathrm{brk}}(t)\right) \omega_{2}(t)+P_{\text {aux }} \\
& +B_{\mathrm{b}}\left(P_{\mathrm{b}}, E_{\mathrm{b}}, n_{\mathrm{b}}\right),
\end{aligned}
$$

problem (36) can be solved efficiently, as a convex second order cone program.

With the known engine on/off signals, the entire convex optimization problem is fully summarized in Table 2.

When solving problem (33) it was here considered that the maps $\tilde{P}_{\mathrm{f}}, \tilde{P}_{\text {cmin }}, \tilde{P}_{\text {cmax }}$ are obtained for all combinations of

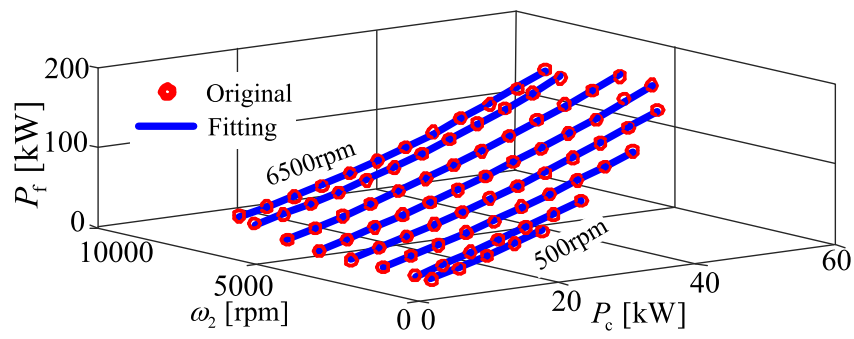

(a) $T_{\mathrm{d}}=50 \mathrm{Nm}$.

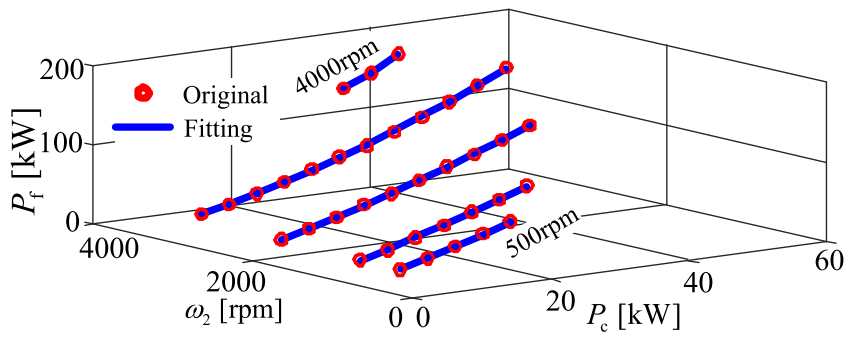

(b) $T_{\mathrm{d}}=250 \mathrm{Nm}$.

Fig. 7. Fuel power of the compound unit when operated at the optimal engine speed. The fuel power is a function of EM2 speed, compound power and demanded torque. The top and bottom plot show the fuel power for two different values of demanded torque. The circles represent the grid points at which the fuel power map is calculated, while the lines show the fitted model.

$\omega_{2}, T_{\mathrm{d}}$ that could represent any realistic driving cycle. However, if the powertrain is to be evaluated only on a specific driving cycle, it is sufficient to only calculate the maps at those $\left(\omega_{2}, T_{\mathrm{d}}\right)$ that are requested by the driving cycle. This could significantly speed up the process of maps generation.

\subsection{Heuristic engine on/off control and varying vehicle mass}

Since convex optimization is not able to solve mixed-integer problem, optimization of $e_{\text {on }}$ has not been directly included in the optimization problem. Instead, the findings in [21] are used to iteratively optimize the engine on/off. It has been shown in [21] that for an HEV powertrain in a series architecture, there exists a constant power threshold $P_{\text {th }}$, for which the optimal engine on/off sequence satisfies

$$
e_{\mathrm{on}}(t)^{*}= \begin{cases}1, & \omega_{2}(t) T_{\mathrm{d}}(t) \geq P_{\mathrm{th}} \\ 0, & \text { otherwise }\end{cases}
$$

when battery open circuit voltage is constant and battery energy limits are not activated along the horizon. In general, battery energy limits may get activated, in which case the engine on/off sequence obtained by (39) may give a suboptimal solution. However, it has been observed in $[22,24]$ that the optimization error is typically small, below $1 \%$.

In a hybrid mode, the EVT powertrain operates similarly to a series powertrain, with the compound unit resembling the engine-generator unit in a series powertrain. 


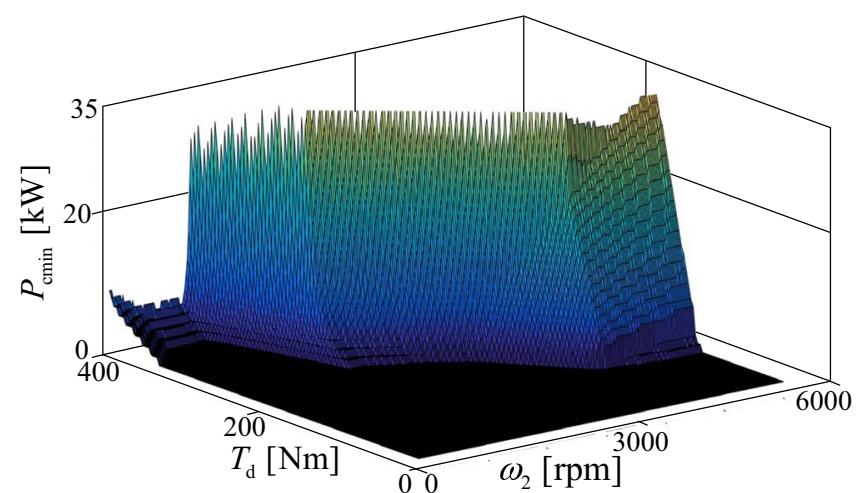

(a) Low limit of compound power.

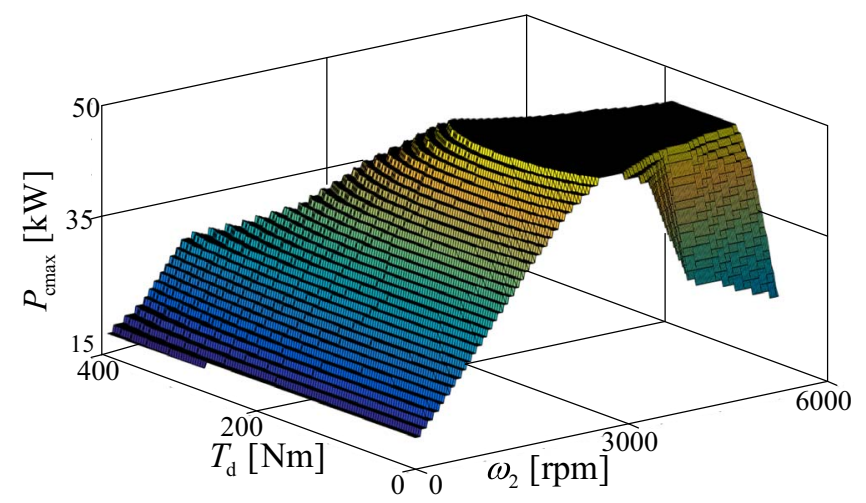

(b) High limit of compound power.

Fig. 8. Limits of the compound unit output $P_{\mathrm{c}}$ when operating at the optimal engine speed. The limits are a function of demanded torque and EM2 speed. (a) Low limit of compound power. (b) High limit of compound power.

Therefore, the engine on/off sequence in the EVT powertrain is found from (39), by obtaining an appropriate power threshold. The optimal value of $P_{\text {th }}$ is tuned by iteratively solving the convex optimization problem, and using, e.g. bisection, to adjust the next value of $P_{\text {th }}[24,26]$. Another presumption of the convex optimization in Table 2 is that the vehicle mass will not change with the battery size. Indeed, if vehicle mass was allowed to change with battery size according to (4), then the fuel power (34) and the power limits (35) of the compound unit, would depend not only on the deterministic disturbances $\omega_{2}, T_{\mathrm{d}}$, but also on the battery size $n_{\mathrm{b}}$. This would require convexity analyses in a four dimensional space and may complicate the derivation of a convex expression as in (37). Instead, to account for changes in battery mass we adopt an iterative method.

First, battery mass is set to an initial value, the required coefficients $k_{\mathrm{i}}$ in (37) are obtained and convex optimization is run to get the optimum battery size. If the mass of the optimal battery size is not the same as the initial value, the procedure is then repeated by iteratively updating the initial battery mass with the latest obtained battery size.

The iterative search for the optimum engine on/off threshold $P_{\text {th }}$, together with the iterative updating of the battery mass is depicted in Figure 9.
Table 2. Convex optimization problem of the EVT powertrain.

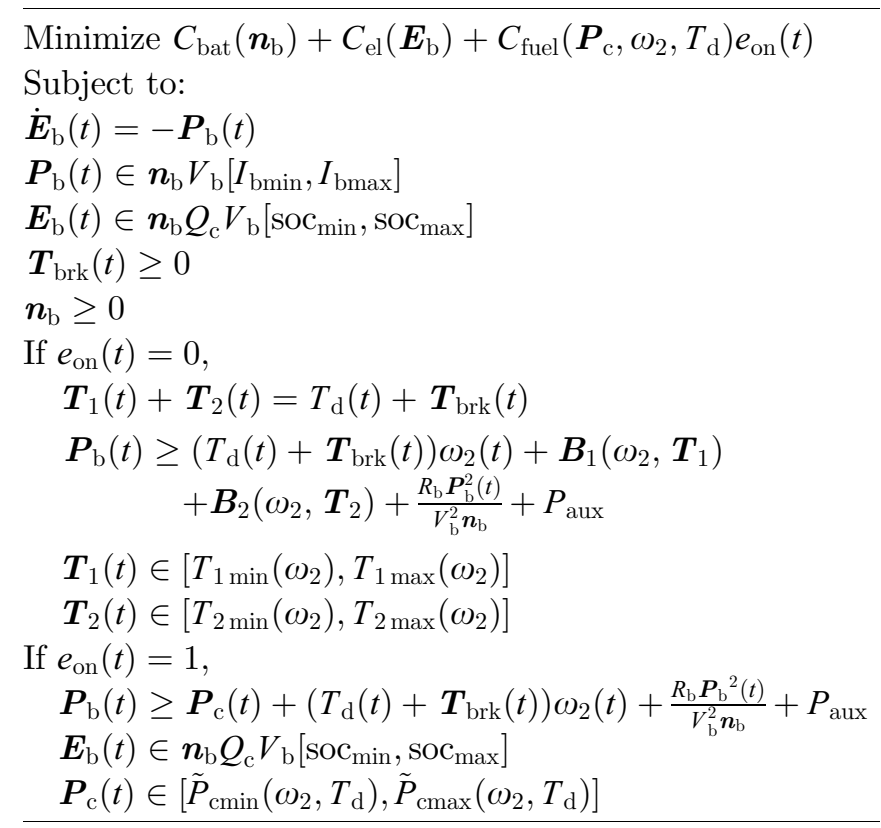

\section{Numerical results}

In this section, simulation results are discussed of the studied optimization problem. To investigate the optimality of the solution, the results are compared with those obtained with DP. The optimization results as well as the algorithm sensitivity to battery mass are discussed. Different battery discharge strategies are compared for the EVT plug-in powertrain. Finally, the advantage is discussed of using a dual EM instead of a single EM when driving in pure electric mode.

The optimization problem is solved by SDPT3 solver and with the CVX toolbox [32].

\subsection{Validation with dynamic programming}

To verify the accuracy of the proposed Convex Programming $(\mathrm{CP})$, the optimality of the results is compared to those obtained from DP. In order to reduce the computational effort that DP would need to solve the benchmark problem, only the optimal energy management of the powertrain over the same driving cycle is compared. The battery size is fixed to $3 \mathrm{~kW}$ and the initial and final battery "soc" values are not free, but fixed to a certain value. Furthermore, to investigate the possible error caused by discretization and second order approximation involved in the compound unit generation, the same heuristic engine on/off control strategy is applied both in DP and in CP.

As shown in Figure 10, the optimal soc trajectory acquired from $\mathrm{CP}$ is almost the same as that acquired from DP, suggesting that the energy management optimized by the CP achieves good accuracy. Also, the fuel consumption calculated by the $\mathrm{CP}$ and the DP over the driving cycle is $3.145 \mathrm{~L} / 100 \mathrm{~km}$ (74.806 mpg) and $3.157 \mathrm{~L} / 100 \mathrm{~km}$ 


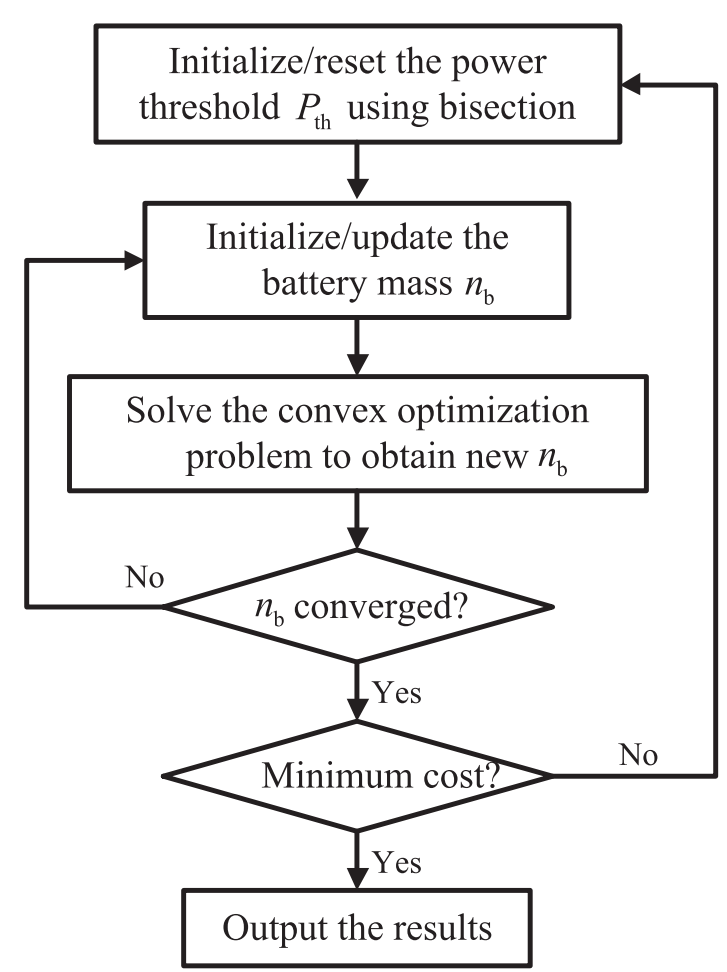

Fig. 9. Iterative procedure for solving the problem of optimal battery sizing and control of an EVT PHEV powertrain. For a given power threshold $P_{\text {th }}$ the engine on/off sequence is decided. Then, for a given on/off sequence, the problem in Table 2 is solved assuming a constant vehicle mass. After optimal battery size $n_{\mathrm{b}}$ is obtained, the vehicle mass is updated and the problem is solved repeatedly until vehicle mass stops changing. The search for the optimal threshold $P_{\mathrm{th}}$ then continues by repeating the entire process.

(74.522 mpg), respectively, showing that the optimization error is within $0.38 \%$.

\subsection{Varying vehicle mass with battery size}

In the $\mathrm{CP}$, the control strategy is optimized simultaneously with the battery sizing, on the assumption that vehicle mass will not change with the battery size. However, the vehicle mass varies with the battery size. To eliminate the error caused by the fixed vehicle mass, we adopt the iterative procedure in Figure 9. The results are shown in Figure 11.

It could be seen that the battery mass converges very quickly during the iterative procedure, regardless of the initially selected value. In fact, the CP needs only $2-3$ iterations to converge to the optimum. This is mainly due to the fact that the battery mass accounts for a relatively small portion of the total vehicle mass.

\subsection{Comparison between different battery discharge strategies}

Aside from the optimization methods, EMS for PHEVs are typically classified into Charge-Depleting-ChargeSustaining (CDCS) and blended control, depending on

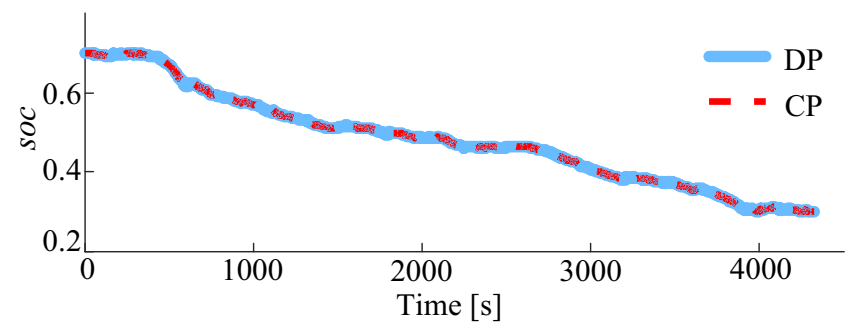

Fig. 10. Comparison of the optimal battery soc trajectory over the driving cycle, between Dynamic Programming (DP) and Convex Programming (CP). The trajectories completely overlap, showing that both algorithms point to the same optimal solution.

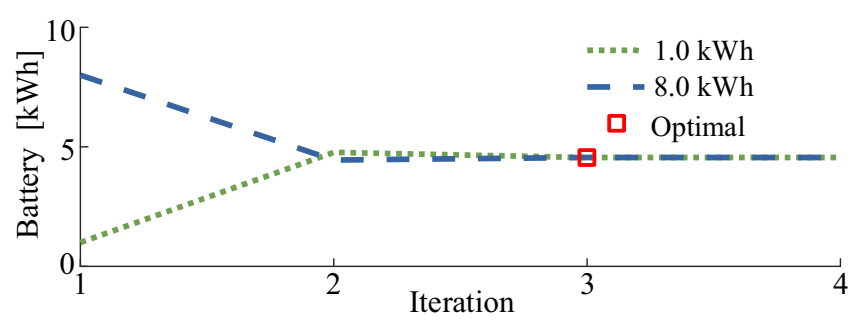

Fig. 11. Convergence of the optimal battery capacity in $\mathrm{kW}$ with different initial battery mass. The battery size is initialized to $1 \mathrm{~kW}$, or $8 \mathrm{~kW}$, respectively. After $2-3$ iterations the battery size converges to the optimal value, depicted by a square marker.

the way the battery is discharged [14]. Implementing the different plug-in HEV control strategies may result in different control schemes and different battery sizes. The CP described in this paper aims for a globally optimal battery discharge strategy, which in this case corresponds to the blended strategy. However, it is of interest to investigate how the CDCS discharge strategy affects the optimal battery sizing. In this part, the optimal results of the blended control strategy are compared with those of the CDCS control strategy.

To calculate the optimal results of the CDCS strategy, the CP previously described can still be used, only with slight modifications. CDCS requires the time instance to be known beforehand, when the algorithm switches from Charge Depleting (CD) to Charge Sustaining (CS) operation. This could be achieved in two different ways. Either the CP could be iteratively solved for different CD durations, or for different battery sizes. Here, the later approach is adopted, by gridding the battery size and running CP to optimize the EMS for each fixed battery size. To find out the switching point from $\mathrm{CD}$ to $\mathrm{CS}$, the torque distribution between EM1 and EM2 is optimized along the driving cycle until the battery soc reaches the lower boundary. Then, the optimal split between the battery and ICE power is calculated from the switching point to the end of the cycle, with the limit $\mathrm{soc} \geq \mathrm{soc}_{\mathrm{min}}$. The initial and final soc values in both discharge strategies are fixed to $\mathrm{soc}_{\max }$ and $\mathrm{Soc}_{\mathrm{min}}$, respectively. The optimal control trajectories of CDCS and blended strategies are shown in Figure 12. 


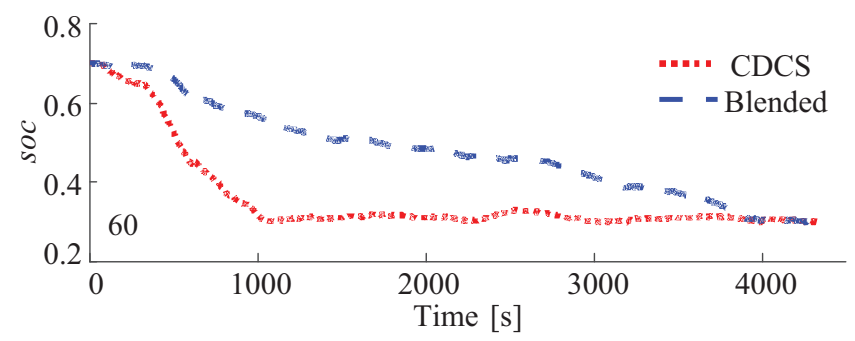

Fig. 12. Optimal soc trajectory with Charge-Depleting-ChargeSustaining (CDCS) and blended battery discharge strategies.

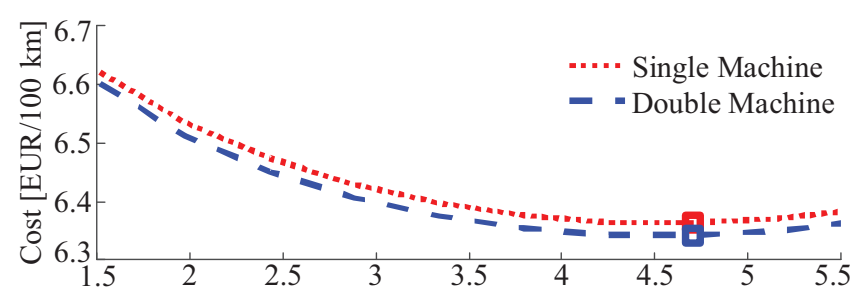

(a)

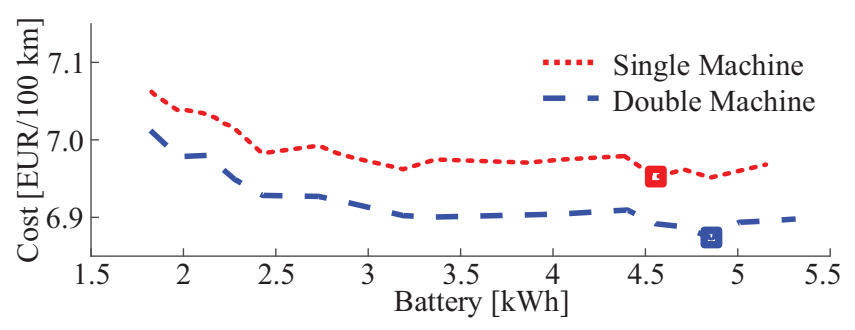

(b)

Fig. 13. Optimal cost for different battery sizes and discharge strategies. The plots depict two optimal results, for the cases where only EM2, or both EM1 and EM2 propel the vehicle in pure electric mode. In the plots, the optimum battery size is denoted by a square marker. (a) Blended battery discharge strategy. (b) CDCS battery discharge strategy.

As shown in Figure 13a, with blended strategy the powertrain would achieve minimum optimization cost at battery size of $4.544 \mathrm{~kW}$, for both single machine drive and double machines drive in pure electric mode. It also suggests that the cost improvement by utilizing double machines drive in blended strategy is minor, only $0.45 \%$. This could be explained by investigating the total driving energy circulated in pure electric mode. In our case, the optimal threshold to turn the engine on is relatively small, around $10 \mathrm{~kW}$, implying that the vehicle is mainly driven in hybrid mode. Moreover, the average speed of the daily driving cycle is relatively high and thus the average power demanded by the driving cycle is well above the optimal on/off threshold. Therefore, since the total driving energy spent in pure electric mode is small, the efficiency improvement from using the double machine is also small.

Optimization results of the CDCS strategy are shown in Figure 13b. The optimal battery size, when only EM2 is used in pure electric mode, is $4.554 \mathrm{~kW}$, while for a double machine drive is $4.858 \mathrm{~kW}$. In this case, the optimal cost

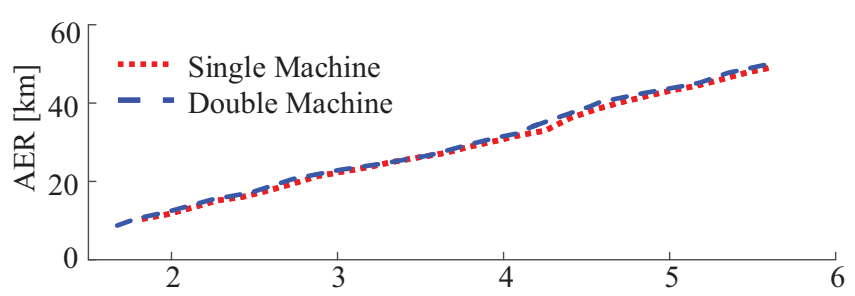

(a)

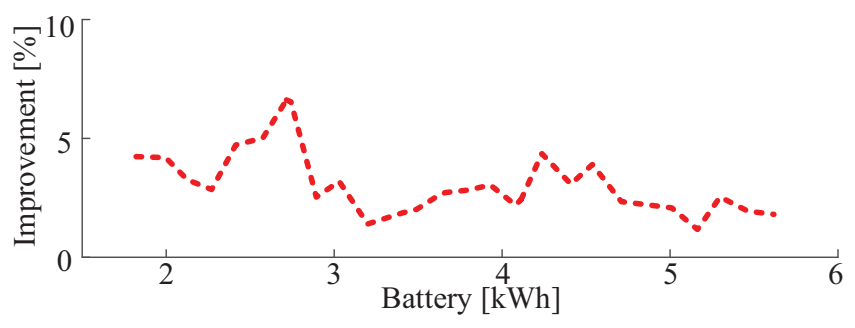

(b)

Fig. 14. All Electric Range (AER) in charge depleting operation. The lower plot depicts the improvement in electric range due to the usage of two EMs for electric propulsion. (a) AER for different battery sizes. (b) AER improvement when using two EMs for propulsion.

from using double machine drive in pure electric mode is reduced by $1.7 \%$, compared to the case when only EM2 propels the vehicle. The reason for the significant improvement is that the total driving time in pure electric mode in CDCS over the driving cycle is longer than that of the blended strategy. With CDCS strategy, the powertrain would deplete the battery energy in the CD phase (namely, the all electric range), so the advantage of double machines drive is more significant.

In Figure 14, the All Electric Range (AER) is depicted in CD operation. The plots show that by using two EMs for electric propulsion, an extra electric range can be obtained, compared to a single machine drive. The improvement of AER performance with different battery sizes is presented in Figure 14b.

\section{Conclusion and future work}

In this paper, convex modeling steps are introduced to simultaneously optimize battery sizing and energy management of plug-in HEV EVT powertrains. For a given engine on/off control sequence, it is shown that both pure electric mode, when engine is off, and hybrid mode, when engine is on, can be modeled as convex programs. In order to convexify the problem in hybrid mode, it is shown that the ICE, EM1 and EM2 can be regarded as a compound unit, whose optimal operating speed can be found independently of the battery discharge and sizing problem. The resulting convex problem is a second order cone program that can be solved efficiently with publicly available solvers.

An investigation is provided where optimization results from convex optimization are compared to those obtained with DP. The results show that for a given engine on/off 
sequence and battery size, the proposed method provides the globally optimal power split control. Then, the convex optimization method is used to compare the optimal cost when using the CDCS and blended battery discharge strategy. The advantage of using double machines drive over a single machine drive in pure electric mode, is presented and discussed.

Due to the efficient computation ability of the convex optimization, future research may focus on configuring the optimization method for real-time control onboard the vehicle. Future studies may also focus on building a synergy between convex optimization and DP, such that optimal engine on/off and power split control can be co-optimized by the two different algorithms.

Acknowledgments. This research is supported by the National Key Research and Development Program of China (2017YFB0102400).

\section{References}

1 Wu G., Zhang X., Dong Z. (2015) Powertrain architectures of electrified vehicles: Review, classification and comparison, J. Franklin Inst. - Eng. Appl. Math. 352, 2, 425-448.

2 Hoeijmakers M., Ferreira J. (2006) The electric variable transmission, IEEE Trans. Ind. Appl. 42, 4, 1092-1100.

3 Vinot E., Trigui R., Cheng Y., Espanet C., Bouscayrol A., Reinbold V. (2014) Improvement of an EVT-based HEV using dynamic programming, IEEE Trans. Veh. Technol. 63, 1, 40-50.

4 Cheng Y., Trigui R., Espanet C., Bouscayrol A., Cui S. (2011) Specifications and design of a PM electric variable transmission for Toyota Prius II, IEEE Trans. Veh. Technol. 60, 9, 4106-4114.

5 Cheng Y., Cui S., Song L., Chan C. (2007) The study of the operation modes and control strategies of an advanced electromechanical converter for automobiles, IEEE Trans. Magn. 43, 1, 430-433.

6 Zhang X., Li C., Kum D., Peng H. (2012) Prius ${ }^{+}$and Volt ${ }^{-}$: configuration analysis of power-split hybrid vehicles with a single planetary gear, IEEE Trans. Veh. Technol. 61, 8, 3544-3552.

7 Padmarajan B., McGordon A., Jennings P. (2016) Blended rule-based energy management for PHEV: System structure and strategy, IEEE Trans. Veh. Technol. 65, 10, 8757-8762.

8 Trovao P., Pereirinha G., Jorge M., Antunes C. (2013) A multi-level energy management system for multi-source electric vehicles - An integrated rule-based meta-heuristic approach, Appl. Energy 105, 304-318.

9 Peng J., He H., Xiong R. (2017) Rule based energy management strategy for a series-parallel plug-in hybrid electric bus optimized by dynamic programming, Appl. Energy 185, 2, 1633-1643.

10 Patil R., Filipi Z., Fathy H. (2014) Comparison of supervisory control strategies for series plug-in hybrid electric vehicle powertrains through dynamic programming, IEEE Trans. Control Syst. Technol. 22, 2, 502-509.

11 Viktor L., Johannesson L., Bo Egardt, Patil R. (2015) Analytic solutions to the dynamic programming subproblem in hybrid vehicle energy management, IEEE Trans. Veh. Technol. 64, 4, 1458-1467.
12 Kim N., Cha S., Peng H. (2011) Optimal control of hybrid electric vehicles based on Pontryagin's minimum principle, IEEE Trans. Control Syst. Technol. 19, 5, 1279-1287.

13 Zou Y., Liu T., Sun F., Peng H. (2013) Comparative study of dynamic programming and Pontryagin's minimum principle, Energies 6, 4, 2305-2318.

14 Moura J., Callaway S., Fathy K., Stein Jeffrey L. (2010) Tradeoffs between battery energy capacity and stochastic optimal power management in plug-in hybrid electric vehicles, J. Power Sources 195, 9, 2979-2988.

15 Bellman R. (2013) Dynamic programming, Dover Publications.

16 Johannesson L., Pettersson S., Egardt B. (2009) Approximate dynamic programming applied to a four quadrant transducer series-parallel hybrid electric bus, 2009 European Control Conference, pp. 4846-5485.

17 Hou C., Ouyang M., Xu L., Wang H. (2014) Approximate Pontryagin's minimum principle applied to the energy management of plug-in hybrid electric vehicles, Appl. Energy 115, 174-189.

18 Onori S., Tribioli L. (2015) Adaptive Pontryagin's Minimum Principle supervisory controller design for the plug-in hybrid GM Chevrolet Volt, Appl. Energy 147, 224-234.

19 Ngo V., Hofman T., Steinbuch M., Serrarens A. (2012) Optimal control of the gearshift command for hybrid electric vehicles, IEEE Trans. Veh. Technol. 61, 8, 3531-3543.

20 Egardt B., Murgovski N., Pourabdollah M., Johannesson L. (2014) Electromobility studies based on convex optimization: Design and control issues regarding vehicle electrification, IEEE Trans. Control Syst. Technol. 34, 2, 32-49.

21 Elbert P., Nüesch T., Ritter A., Murgovski N., Guzzella L. (2014) Engine on/off control for the energy management of a serial hybrid electric bus via convex optimization, IEEE Trans. Veh. Technol. 63, 8, 3549-3559.

22 Murgovski N., Johannesson L., Sjöberg J. (2013) Engine on/off control for dimensioning hybrid electric powertrains via convex optimization, IEEE Trans. Veh. Technol. 62, 7, 2949-2962.

23 Murgovski N., Johannesson L., Egardt B. (2014) Optimal battery dimensioning and control of a CVT PHEV powertrain, IEEE Trans. Veh. Technol. 63, 5, 2151-2161.

24 Murgovski N., Johannesson L., Sjöberg J., Egardt B. (2012) Component sizing of a plug-in hybrid electric powertrain via convex optimization, Mechatronics 22, 1, 106-120.

25 Pourabdollah M., Murgovski N., Grauers A., Egardt B. (2013) Optimal sizing of a parallel PHEV powertrain, IEEE Trans. Veh. Technol. 62, 6, 2469-2480.

$26 \mathrm{Hu}$ X., Moura S., Murgovski N., Egardt B., Cao D. (2016) Integrated optimization of battery sizing, charging, and power management in plug-in hybrid electric vehicles, IEEE Trans. Control Syst. Technol. 24, 3, 1036-1043.

$27 \mathrm{Hu}$ X., Murgovski N., Johannesson L., Egardt B. (2013) Energy efficiency analysis of a series plug-in hybrid electric bus with different energy management strategies and battery sizes, Appl. Energy 111, 1001-1009.

$28 \mathrm{Hu}$ X., Lar Johannesson, Murgovski N., Egardt B. (2015) Longevity-conscious dimensioning and power management of the hybrid energy storage system in a fuel cell hybrid electric bus, Appl. Energy 137, 913-914.

29 Nüeesch T., Elbert P., Flankl M., Onder C., Guzzella L. (2014) Convex optimization for the energy management of hybrid electric vehicles considering engine start and gearshift costs, Energies 7, 2, 834-856. 
30 Johannesson L., Pettersson S., Egardt B. (2014) Computationally efficient energy management of a planetary gear hybrid electric vehicle, IFAC Proc. Vol., pp. 4831-4836.

31 Murgovski N., Johannesson L., Hu X., Bo E, Sjöberg J. (2015) Convex relaxations in the optimal control of electrified vehicles, 2015 American Control Conference, pp. 2292-2298.

32 Grant M., Boyd S. (2014) CVX: Matlab Software for Disciplined Convex Programming, version 2.1. Available at http://cvxr.com/cvx/.

\section{Appendix}

The vehicle powertrain parameters are listed in Table A1.

Table A1. Parameters of the powertrain.

\begin{tabular}{ll}
\hline Parameter & Value \\
\hline Rolling resistance & $f=0.005$ \\
Aerodynamic drag coefficient & $C_{\mathrm{d}}=0.3$ \\
Vehicle mass & $m_{\mathrm{g}}=1350 \mathrm{~kg}$ \\
Frontal area & $A_{\mathrm{f}}=1.76 \mathrm{~m}^{2}$ \\
Air density & $\rho=1.30 \mathrm{~kg} / \mathrm{m}^{3}$ \\
Wheel radius & $r=0.237 \mathrm{~m}$ \\
Battery cell's capacity & $Q_{\mathrm{b}}=2.3 \mathrm{Ah}$ \\
Battery cell's open-circuit voltage & $V_{\mathrm{b}}=3.3 \mathrm{~V}$ \\
Battery cell's internal resistance & $R_{\mathrm{b}}=0.001$ \\
Maximum charge current & $I_{\mathrm{bmax}}=120 \mathrm{~A}$ \\
Maximum discharge current & $I_{\mathrm{bmin}}=-70 \mathrm{~A}$ \\
Maximum soc & $\mathrm{soc}_{\max }=0.7$ \\
Minimum soc & $\mathrm{soc}_{\min }=0.3$ \\
ICE idling speed & $\omega_{\mathrm{emin}}=970 \mathrm{rpm}$ \\
ICE specification $\left(T_{\text {emax }}, \omega_{\text {emax }}\right)$ & $110 \mathrm{Nm}, 4790 \mathrm{rpm}$ \\
EM1 specification $\left(T_{1 \max }, \omega_{1 \max }\right)$ & $120 \mathrm{Nm}, 6400 \mathrm{rpm}$ \\
EM2 specification $\left(T_{2 \max }, \omega_{2 \max }\right)$ & $300 \mathrm{Nm}, 7000 \mathrm{rpm}$ \\
\hline
\end{tabular}

